\title{
Absence of a boron isotope effect in the magnetic penetration depth of $\mathrm{MgB}_{2}$
}

\author{
D. Di Castro, ${ }^{1, *}$ M. Angst, ${ }^{1}$ D. G. Eshchenko, ${ }^{1,2}$ R. Khasanov, ${ }^{1,2}$ J. Roos, ${ }^{1}$ I. M. Savić, ${ }^{3}$ A. Shengelaya, ${ }^{1}$ S. L. Bud'ko, ${ }^{4}$ \\ P. C. Canfield, ${ }^{4}$ K. Conder, ${ }^{5}$ J. Karpinski, ${ }^{6}$ S. M. Kazakov, ${ }^{6}$ R. A. Ribeiro, ${ }^{4}$ and H. Keller ${ }^{1}$ \\ ${ }^{1}$ Physik-Institut der Universität Zürich, CH-8057 Zürich, Switzerland \\ ${ }^{2}$ Paul Scherrer Institute, CH-5232 Villigen PSI, Switzerland \\ ${ }^{3}$ Faculty of Physics, University of Belgrade, 11001 Belgrade, Yugoslavia \\ ${ }^{4}$ Ames Laboratory and Department of Physics and Astronomy, Iowa State University, Ames, Iowa 50011, USA \\ ${ }^{5}$ Laboratory for Neutron Scattering, ETH Zürich and PSI Villigen, CH-5232 Villigen PSI, Switzerland \\ ${ }^{6}$ Solid State Physics Laboratory, ETH, CH-8093 Zürich, Switzerland
}

(Received 3 May 2004; published 30 July 2004)

\begin{abstract}
The magnetic penetration depth $\lambda(0)$ in polycrystalline $\mathrm{MgB}_{2}$ for different boron isotopes $\left({ }^{10} \mathrm{~B} /{ }^{11} \mathrm{~B}\right)$ was investigated by transverse field muon spin rotation. No boron isotope effect on the penetration depth $\lambda(0)$ was found within experimental error: $\Delta \lambda(0) / \lambda(0)=0.8(8) \%$, suggesting that $\mathrm{MgB}_{2}$ is an adiabaic superconductor. This is in contrast to the substantial oxygen isotope effect on $\lambda(0)$ observed in cuprate high-temperature superconductors.
\end{abstract}

DOI: 10.1103/PhysRevB.70.014519

PACS number(s): 74.70.Ad, 71.38. $-\mathrm{k}, 76.75 .+\mathrm{i}, 82.20 . \mathrm{Tr}$

Since the discovery of superconductivity with transition temperature $T_{c} \approx 39 \mathrm{~K}$ in the binary intermetallic compound $\mathrm{MgB}_{2},{ }^{1}$ a large number of experimental and theoretical investigations were performed in order to explain the mechanism and the origin of its remarkably high transition temperature. Experiments were done revealing the important role played by the lattice excitations in this material. ${ }^{2-5}$ In particular, the substitution of the ${ }^{11} \mathrm{~B}$ with ${ }^{10} \mathrm{~B}$ has been demonstrated to shift $T_{c}$ to higher temperatures, ${ }^{2,3}$ as expected for a phonon mediated pairing mechanism.

However, $\mathrm{MgB}_{2}$ differs from conventional superconductors in several important aspects, including, for instance, the unusually high $T_{c}$ and the anomalous specific heat. ${ }^{6}$ Calculations ${ }^{7,8}$ based on the Eliashberg formalism support the experimental results, ${ }^{6,9-11}$ revealing $\mathrm{MgB}_{2}$ to be a twoband superconductor with two superconducting gaps of different size, the larger one originating from a $2 \mathrm{D} \sigma$-band and the smaller one from a 3D $\pi$-band. The electronic $\sigma$-states are confined to the boron planes and couple strongly to the in-plane vibration of the boron atoms ( $E_{2 g}$ phonon mode). This strong pairing, confined only to parts of the Fermi surface, is the principal contribution responsible for superconductivity and mainly determines $T_{c}$. The $\pi$-states on the remaining parts of the Fermi surface form much weaker pairs. The double-gap structure explains most of the unusual physical properties of $\mathrm{MgB}_{2}$, such as the high critical temperature, the total $T_{c}$ isotope-effect coefficient $\left(\alpha \approx 0.32^{3}\right)$, the temperature dependent specific heat, ${ }^{6}$ tunneling, ${ }^{10}$ and upper critical field anisotropy $H_{c 2}^{\| a b} / H_{c 2}^{\| c}{ }^{12}$

An interesting point to be clarified concerns the nature of the electron-lattice coupling. It was proposed ${ }^{13-15}$ that $\mathrm{MgB}_{2}$ is a nonadiabatic superconductor. Alexandrov ${ }^{13}$ suggested that, because of the large coupling strength of the electrons to the $E_{2 g}$ phonon mode, the many-electron system is unstable and breaks down into a small polaron system, similar to the cuprate high temperature superconductors (HTSC), where the charge carriers are trapped by local lattice distortions. Cappelluti et al. ${ }^{14}$ proposed that the small value of the
Fermi energy $E_{F}$ of the $\sigma$ bands relative to the phonon energy $\omega_{\text {ph }}$ violates the adiabatic assumption $\left(\omega_{\mathrm{ph}} \ll E_{F}\right)$, opening up a nonadiabatic channel that enhances $T_{c}$. Both these nonadiabatic models ${ }^{13,14}$ explicitly predict, but not quote, a boron isotope effect (BIE) on the carrier effective mass $m^{*}$ in $\mathrm{MgB}_{2}$. Zhao ${ }^{15}$ proposed an unconventional phonon mediated mechanism for superconductivity, predicting a boron isotope effect on the inverse squared magnetic penetration depth $\lambda^{-2}$ of $4 \%$. Similar models ${ }^{16-18}$ were already used to explain the large oxygen isotope effect (OIE) on the magnetic field penetration depth $\lambda$, a physical quantity directly related to the charge carrier effective mass $m^{*}$, observed in HTSC. ${ }^{19-26}$ The nonadiabatic models are in contrast to the conventional theory of superconductivity (Migdal adiabatic approximation), in which the density of states at the Fermi level, the electron-phonon coupling constant, and the effective supercarrier mass $m^{*}$ are all independent of the mass $M$ of the lattice atoms.

Here, a muon spin rotation $(\mu \mathrm{SR})$ study of the magnetic penetration depth $\lambda(0)$ in polycrystalline $\mathrm{MgB}_{2}$ for different boron isotopes $\left({ }^{10} \mathrm{~B} /{ }^{11} \mathrm{~B}\right)$ is reported. $\mu \mathrm{SR}$ is a powerful microscopic tool to measure the magnetic penetration depth $\lambda .{ }^{27}$ Indeed, in a polycrystalline type II superconductor with a perfect vortex lattice (VL) the average magnetic penetration depth $\lambda$ can be extracted from the muon-spin depolarization rate $\sigma(T) \propto \lambda^{-2}(T) .{ }^{27}$ In our measurement, no BIE on $\lambda(0)$ was observed within experimental error $[\Delta \lambda(0) / \lambda(0)$ $=0.8(8) \%]$, in contrast to the substantial OIE observed in cuprate HTSC. ${ }^{19-26}$ Our results imply that polaronic or nonadiabatic effects in $\mathrm{MgB}_{2}$ are absent or negligibly small.

To our knowledge, our experiments also provide for the first time a direct experimental evidence for the absence of an isotope effect on the penetration depth in a conventional superconductor. Only in a rather indirect way, it was shown ${ }^{28}$ by critical field measurements, that in the conventional strong-coupling superconductor lead there is no isotope effect on the coefficient of the normal electronic specific heat. This confirms the validity of the adiabatic approximation in this system. 


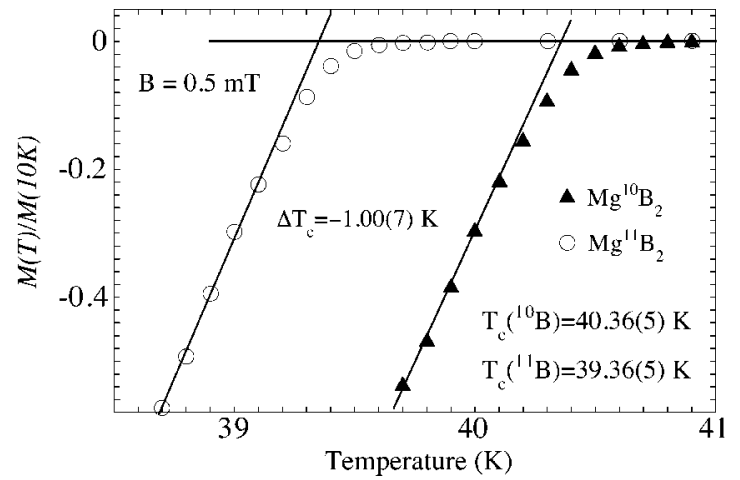

FIG. 1. Normalized field cooled $(0.5 \mathrm{mT})$ magnetization as a function of temperature for $\mathrm{Mg}^{10} \mathrm{~B}_{2}$ and $\mathrm{Mg}^{11} \mathrm{~B}_{2}$ samples.

The $\mu \mathrm{SR}$ experiments were performed on two polycrystalline $\mathrm{MgB}_{2}$ samples containing ${ }^{11} \mathrm{~B}\left(\mathrm{Mg}^{11} \mathrm{~B}_{2}\right)$ and ${ }^{10} \mathrm{~B}\left(\mathrm{Mg}^{10} \mathrm{~B}_{2}\right)$. Full details of the sample synthesis are given in Refs. 2 and 29. In brief, the two samples were synthesized using elemental $\mathrm{Mg}$ (99.9\% pure in lump form) and isotopically pure boron $(99.95 \%$ chemical purity, $99.5 \%$ isotope purity, $<100$ mesh) combined in a sealed Ta tube in a stoichiometric ratio. The Ta tube was then sealed in a quartz ampoule, placed in a $950^{\circ} \mathrm{C}$ box furnace for $24 \mathrm{~h}$, and then removed and allowed to cool to room temperature.

To examine the quality of the samples low field $(0.5 \mathrm{mT}$, field-cooled) magnetization measurements were performed using a commercial Superconducting Quantum Interference Device. Figure 1 shows the temperature dependence of the magnetization for the $\operatorname{Mg}^{11} \mathrm{~B}_{2}$ and $\mathrm{Mg}^{10} \mathrm{~B}_{2}$ samples in the vicinity of $T_{c}$. The high quality of the two samples is revealed by the sharp transition and the high $T_{c}$ extracted from the intercept of the linear extrapolations (Fig. 1): $T_{c}\left({ }^{10} \mathrm{~B}\right.$ ) $=40.36(5) \mathrm{K}, T_{c}\left({ }^{11} \mathrm{~B}\right)=39.36(5) \mathrm{K}$. There is a clear isotope shift of $\Delta T_{c}=T_{c}\left({ }^{11} \mathrm{~B}\right)-T_{c}\left({ }^{10} \mathrm{~B}\right)=-1.00(7) \mathrm{K}$. The corresponding isotope effect coefficient $\alpha_{B}=-d \ln \left(T_{c}\right) / d \ln \left(M_{B}\right)$ $=0.29$ (2) (enrichment corrected) is in good agreement with previous results.,3

The transverse-field $\mu \mathrm{SR}$ experiments were performed at the Paul Scherrer Institute (PSI), Switzerland, using the $\pi \mathrm{M} 3$ $\mu \mathrm{SR}$ facility. The samples used for the magnetization measurements (see Fig. 1) were pressed in disk-shaped pellets with $10 \mathrm{~mm}$ diameter and $3 \mathrm{~mm}$ thickness and cooled in an external magnetic field $B_{\text {ext }}$ perpendicular to the muon spin polarization from well above $T_{c}$ to temperatures lower than $T_{c}$. The measurements were taken in a field of $B_{\mathrm{ext}}=0.6 \mathrm{~T}$ (the highest available at PSI), high enough to avoid pinning induced distortion of the VL. ${ }^{30-32}$ As shown in Fig. 2 for $\mathrm{Mg}^{11} \mathrm{~B}_{2}$ at two different temperatures, the local magnetic field distribution can be very well approximated by a single Gaussian, centered at a field lower than the external one. This again indicates the high quality of the samples and the absence of any normal conducting domains. From the width of the Gaussian field distribution, which is proportional to the muon spin depolarization rate $\sigma$, the penetration depth $\lambda$, that is the length scale of the variation of the magnetic field, can be extracted using the relation $\lambda^{-2} \propto \sigma$.

In Fig. 3, the temperature dependence of $\sigma$ for the $\mathrm{Mg}^{11} \mathrm{~B}_{2}(\bigcirc)$ and $\mathrm{Mg}^{10} \mathrm{~B}_{2}(\boldsymbol{\Delta})$ samples is shown. Below $T_{c}, \sigma$

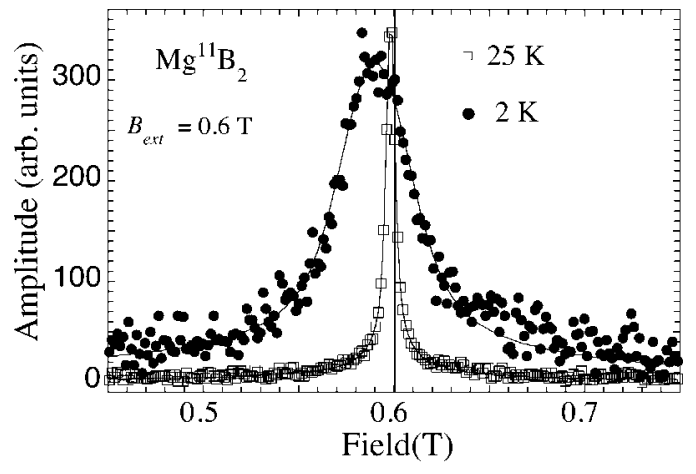

FIG. 2. Local magnetic field distribution, obtained from the Fourier transform of the muon spin precession signal, for $\mathrm{Mg}^{11} \mathrm{~B}_{2}$ at $2(\bullet)$ and $25 \mathrm{~K}(\square)$. Solid lines are Gaussian fits to the experimental data. The vertical solid line indicates the external field of $0.6 \mathrm{~T}$.

for both samples starts to increase and saturates at low temperatures $T \leqslant 6 \mathrm{~K}$, in agreement with previous $\mu \mathrm{SR}$ measurements. ${ }^{31}$ The data for the two samples close to $T_{c}$ show a clear isotope shift of $\Delta T_{c}=-1.2(2) \mathrm{K}$, in agreement with $\Delta T_{c}$ deduced from the low field magnetization measurements (Fig. 1). With decreasing temperature, the values of $\sigma$ for $\mathrm{Mg}^{11} \mathrm{~B}_{2}$ sample are systematically lower than those for the $\mathrm{Mg}^{10} \mathrm{~B}_{2}$ sample. However, at low temperature they merge together, indicating that there is no substantial BIE on $\sigma(0)$.

In order to quantify this observation, we performed fits to the experimental data. It was suggested ${ }^{31,32}$ that for the twogap superconductor $\mathrm{MgB}_{2}$, the temperature dependence of $\sigma$ can be written in the form:

$$
\sigma(T)=\sigma(0)-w \cdot \delta \sigma\left(\Delta_{1}, T\right)-(1-w) \cdot \delta \sigma\left(\Delta_{2}, T\right)
$$

with $\delta \sigma(\Delta, T)=\left[2 \sigma(0) / k_{B} T\right] \int_{0}^{\infty} f(\varepsilon, T) \cdot[1-f(\varepsilon, T)] d \varepsilon$.

Here, $\Delta_{1}$ and $\Delta_{2}$ are the zero temperature large and small gap, respectively, $w$ is the relative contribution of the large gap to $\lambda^{-2}(0)$, and $f(\varepsilon, T)$ is the Fermi distribution. For the temperature dependence of the gaps we used the conven-

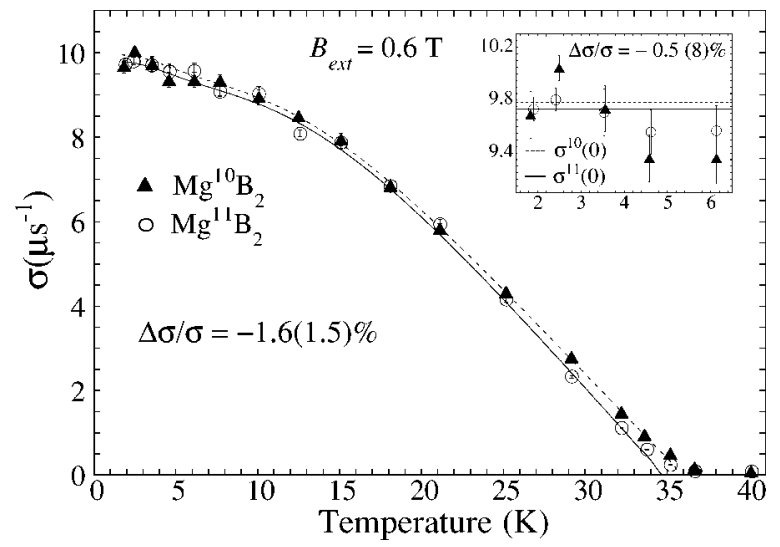

FIG. 3. Temperature dependence of $\sigma$ at $B_{\text {ext }}=0.6 \mathrm{~T}$ for the two isotope samples $\mathrm{Mg}^{10} \mathrm{~B}_{2}(\boldsymbol{\Delta})$ and $\mathrm{Mg}^{11} \mathrm{~B}_{2}(\bigcirc)$. The solid $\left(\mathrm{Mg}^{10} \mathrm{~B}_{2}\right)$ and dotted $\left(\mathrm{Mg}^{11} \mathrm{~B}_{2}\right)$ lines are fits using Eq. (1). Inset: lowtemperature region on a larger scale. The dotted and solid horizontal lines represent the weighed average values of $\sigma(0)$ for $T<7.5 \mathrm{~K}$ for $\mathrm{Mg}^{10} \mathrm{~B}_{2}$ and $\mathrm{Mg}^{11} \mathrm{~B}_{2}$, respectively. 
tional BCS $\Delta(T)$. In order to improve the ratio of the number of data point vs the number of fit parameters, the two gaps and $w$ were considered as common fitting parameters for the two isotope data. As shown by the solid and dotted lines in Fig. 3, the experimental data are well described by Eq. (1). The fit yields: $\sigma(0)^{11} \mathrm{~B}=9.79(10) \mu s^{-1}, \sigma(0){ }^{10} \mathrm{~B}$ $=9.95(11) \mu s^{-1}, w=0.88(2), \Delta_{1}=4.9(1)$, and $\Delta_{2}=1.1(3)$. All these values are in very good agreement with previous $\mu \mathrm{SR}$ measurements performed by us on a natural boron $\mathrm{MgB}_{2}$ sample and by Ohishi $e t$ al. $^{32}$ It is interesting to note that the high value of $w$ implies that only a very small contribution to $\sigma(0)$ originates from the $\pi$-band, in accordance with the experimental finding that the superfluid density in the $\pi$-band is strongly suppressed by an external magnetic field. ${ }^{6,9,10,33-35}$ Below we discuss this issue in more details.

The relative isotope shift of $\sigma(0)$ is

$$
\begin{aligned}
\left(\sigma(0){ }^{11}{ }^{11}-\sigma(0){ }^{10}\right) / \sigma(0) & \equiv \Delta \sigma(0) / \sigma(0)=\Delta \lambda^{-2}(0) / \lambda^{-2}(0) \\
& =-1.6(1.5) \%,
\end{aligned}
$$

corresponding to:

$$
\Delta \lambda(0) / \lambda(0)=0.8(8) \% .
$$

For comparison, we calculated the relative isotope shift using a different and model independent procedure, taking the weighed average of the experimental points for $T<7.5 \mathrm{~K}$ (see inset of Fig. 3), where $\sigma(T)$ saturates. We obtained $\Delta \sigma(0) / \sigma(0)=\Delta \lambda^{-2}(0) / \lambda^{-2}(0)=-0.5(8) \%$. Both the procedures give results compatible with zero BIE on the penetration depth $\lambda(0)$.

Here, it is very important to recall that the two isotope samples used in the experiment were made with the same starting $\mathrm{Mg}$ for both the samples, and with ${ }^{10} \mathrm{~B}$ and ${ }^{11} \mathrm{~B}$ powders of the same mesh size (distribution of grain sizes), and were synthesized under exactly the same conditions. Therefore, we can exclude any influence on $\sigma$ due to different grain size and to a difference in pinning or vortex dynamical effects.

To check the reliability of our results, a second measurement on a set (set B) of samples from different source and preparation technique and with smaller Meissner fraction, was performed in a field of $0.4 \mathrm{~T}$. The results are very similar to the first set (set A) shown above: $\Delta \lambda^{-2}(0) / \lambda^{-2}(0)$ $=-1.5(1.7) \%$ as compared to the above $-1.6(1.5) \%$. This shows that our result is intrinsic for $\mathrm{MgB}_{2}$ and holds for lower fields as well. A summary of the results for both sets of isotope samples is given in Table I. Note that the values of $\sigma(0){ }^{11} \mathrm{~B}$ and $\sigma(0){ }^{10} \mathrm{~B}$ for set $\mathrm{B}$ measured in lower fields are larger than the corresponding values for set $\mathrm{A}$. This is not a consequence of flux lattice pinning or vicinity to the lower critical field $H_{c 1}\left(0.4 \mathrm{~T}\right.$ and $0.6 \mathrm{~T}$ are fields well above $\left.H_{c 1}\right)$, but is due to the field dependence of $\lambda^{-2}$ caused by the suppression of the superfluid density in the $\pi$-band, as shown in several previous works. ${ }^{6,9,10,33-35}$ In particular, Cubitt et al. ${ }^{33}$ by means of small angle neutron scattering, and, more recently, Lyard et al. ${ }^{34}$ and Angst et al.,${ }^{35}$ by means of magnetization measurement, show that the superfluid density $\lambda^{-2}$, rapidly decreases with increasing magnetic field from $0.1 \mathrm{~T}$
TABLE I. Summary of the BIE results for $\sigma(0)$ obtained from the $\mu \mathrm{SR}$ measurements of two sets of isotope samples.

\begin{tabular}{lcccc}
\hline \hline & $\begin{array}{c}\sigma(0)^{10} \mathrm{~B} \\
\left(\mu s^{-1}\right)\end{array}$ & $\begin{array}{c}\sigma(0)^{11} \mathrm{~B} \\
\left(\mu s^{-1}\right)\end{array}$ & \multicolumn{2}{c}{$\Delta \lambda^{-2}(0) / \lambda^{-2}(0)$} \\
\hline Set A & $9.95(11)$ & $9.79(10)$ & $-0.016(15)^{\mathrm{a}}$ & $-0.005(8)^{\mathrm{b}}$ \\
Set B & $12.91(17)$ & $12.69(13)$ & $-0.015(17)^{\mathrm{a}}$ & $-0.016(30)^{\mathrm{b}}$ \\
\hline \hline
\end{tabular}

${ }^{\mathrm{a}}$ From fit using Eq. (1).

${ }^{b}$ From low temperature average (inset Fig. 3).

to $1.2-1.5 \mathrm{~T}$. This field dependence as a result of the two band superconductivity in $\mathrm{MgB}_{2}$ may be likened to, yet is different from, the field dependence due to nonlocal effects as a consequence of the nodes in the gap in cuprate superconductors. In the latter case, the nonlocal effects cause a correction to the zero field penetration depth, ${ }^{36}$ and therefore an isotope effect on the zero field penetration depth should be reflected in an isotope effect on the penetration depth in any field. The only exception would be a masking of a zero field penetration depth isotope effect by an opposite isotope effect on the correction terms, in a weird coincidence. The fact that we obtain no significant isotope effect in two different fields would seem to rule out such a scenario. In the case of $\mathrm{MgB}_{2}$, however, low field measurements simply probe the superfluid density in $\sigma$ and $\pi$ bands together, whereas high field measurements (as done here, particularly in the $0.6 \mathrm{~T}$ measurement) probe the superfluid density in the $\sigma$ bands alone.

We note that we did not attempt to decompose the effective penetration depth $\lambda$ into $\lambda_{a b}$ and $\lambda_{c}$. It was shown $n^{37}$ that in anisotropic polycrystalline samples with large anisotropy factor $\gamma, \lambda$ is mainly determined by the in-plane penetration depth $\lambda_{a b}: \lambda=k \lambda_{a b}$, with $k$ varying with $\gamma$. In $\mathrm{MgB}_{2}$, however, $\gamma$ has a characteristic behavior as a function of temperature and magnetic field (see, for example, Refs. 12 and 33), due to the presence of two distinct bands, but not yet fully understood in a quantitative way. Particularly with a field dependent anisotropy, an accidental cancellation of nonzero isotope effects on $\lambda_{c}$ and $\lambda_{a b}$ both in 0.4 and $0.6 \mathrm{~T}$ can be considered highly unlikely. Therefore, in the rest of the paper we continue to use the effective $\lambda$.

It is interesting to contrast the result given in Eq. (2) with the oxygen isotope effect found in cuprate superconductors. ${ }^{19-26}$ At all doping levels a substantial oxygen isotope effect on $\lambda_{a b}$ was observed, ranging from $2.8(1) \%$, close to optimal doping, ${ }^{25}$ up to about $5(1) \%$ in the underdoped regime. ${ }^{22,24}$ The BIE found here [0.8(8)\%] is well below the values found in cuprates, and rather compatible with zero effect. Moreover this value is also considerably smaller than predicted in Ref. 15.

Theoretically, the zero temperature penetration depth is proportional to a density-of-states weighed average of a tensor involving the Fermi velocities. Detailed calculations within different formalisms have been carried out for $\mathrm{MgB}_{2}$ (see Refs. 38 and 39). For our purpose it is sufficient to use the simpler London approach considering a free electron model and linking $\lambda(0)$ to the superconducting charge carrier density $n_{s}$ and effective mass $m^{*}$, only considering different 
contributions from the $\sigma$ and the $\pi$ bands. There is of course a direct connection between the Fermi velocities and the effective mass (a band average), both of which are not bare quantities, but in general renormalized, e.g., due to coupling with the phonons. The London approach has the advantage of facilitating the comparison with theoretical predictions ${ }^{13,14}$ and results obtained on cuprate superconductors, ${ }^{19-26}$ all of which are formulated within this approach.

Unlike the cuprate superconductors with their extremely short coherence lengths, $\mathrm{MgB}_{2}$ cannot be considered as being in the superclean limit and we need to consider a possible impact of scattering. In a moderately clean superconductor the penetration depth is related to the effective mass $m^{*}$ by the following relation: ${ }^{40}$

$$
1 / \lambda^{2}=\left[\mu_{0} e^{2} / c^{2}\right]\left(n_{s} / m^{*}\right)[1 /(1+\xi / \ell)],
$$

where $n_{s}$ and $m^{*}$ are the superconducting charge carrier density and effective mass, respectively, $\xi$ is the coherence length, and $\ell$ is the mean free path. As already mentioned, the major contribution $(\sim 90 \%)$ to $\lambda^{-2}$ in our experimental conditions comes from the $\sigma$-band. Therefore $n_{s}, m^{*}, \xi$, and $\ell$ in Eq. (3) have to be considered as $\sigma$-band values. It was estimated $^{41,42}$ that in the $\sigma$-band $(\xi / \ell)_{\sigma} \approx 1 / 8$, a value which is close to the clean limit $(\xi / \ell \ll 1)$. Therefore Eq. (3) may be approximated by $1 / \lambda^{2} \approx\left[\mu_{0} e^{2} / c^{2}\right]\left(n_{s} / m^{*}\right)$. A shift in $1 / \lambda^{2}$ due to the isotope substitution is then given by

$$
\frac{\Delta \lambda^{-2}(0)}{\lambda^{-2}(0)}=\frac{\Delta n_{s}}{n_{s}}-\frac{\Delta m^{*}}{m^{*}} .
$$

The contribution from the supercarrier density $n_{s}$ is negligible, as was already experimentally demonstrated in the case of HTSC. ${ }^{20-22}$ Specifically, for $\mathrm{MgB}_{2}$, it can be argued that: (i) by changing the isotope only the mass of the nuclei is changed and not the charge carrier density $n$. Furthermore, $\mathrm{MgB}_{2}$ is a stoichiometric compound; (ii) x-ray diffraction measurements, performed on the samples used for the $\mu \mathrm{SR}$ experiments, showed no substantial difference between the lattice parameters of $\mathrm{Mg}^{11} \mathrm{~B}_{2}$ and $\mathrm{Mg}^{10} \mathrm{~B}_{2}$. This implies that the band structure is not appreciably modified by the isotope substitution. Therefore, assuming $\Delta n_{s} / n_{s} \approx 0$ in Eq. (4) and neglecting the small $\pi$-band contribution, we can estimate the boron isotope effect on the $\sigma$-band effective mass $m_{\sigma}^{*}$ :

$$
\Delta m_{\sigma}^{*} / m_{\sigma}^{*} \approx-\Delta \lambda^{-2}(0) / \lambda^{-2}(0)=1.6(1.5) \% .
$$

Here we have used the value of the relative shift on $\lambda^{-2}(0)$ obtained from the fit to Eq. (1). There is no BIE on the $\sigma$-band effective mass within experimental error.

Our result then suggests that nonadiabatic or polaronic effects in $\mathrm{MgB}_{2}$ are absent or negligibly small, and establishes an upper limit [Eq. (2) and Eq. (5)] to any theoretical prediction of such effects. ${ }^{13-15}$ This conclusion is in contrast to cuprate superconductors, where a substantial oxygen isotope effect on $m^{*}$, well above the upper limit stated here, was observed. ${ }^{19-26}$ Recent magnetization measurements on $\mathrm{MgB}_{2}$ under pressure ${ }^{43}$ show no substantial pressure effect on the magnetic penetration depth $\lambda$ at low temperature, further supporting the main conclusion of the present work.

In summary, $\mu$ SR experiments on polycrystalline $\mathrm{Mg}^{10} \mathrm{~B}_{2}$ and $\mathrm{Mg}^{11} \mathrm{~B}_{2}$ samples revealed no substantial boron isotope effect on the magnetic penetration depth at $T=0 \mathrm{~K}$. From this finding we conclude that there is no substantial BIE on the effective mass $m_{\sigma}^{*}$ of the charge carriers in the $\sigma$ band. This result suggests that $\mathrm{MgB}_{2}$ is a conventional phonon mediated superconductor without nonadiabatic or polaronic effects, in contrast to cuprate superconductors.

This work was partly performed at the Swiss Muon Source $(\mathrm{S} \mu \mathrm{S})$ at the Paul Scherrer Institute (Villigen, Switzerland). We thank D. Herlach and A. Amato for technical assistance during the $\mu \mathrm{SR}$ experiments at the Paul Scherrer Institute and T. Schneider for useful discussions. This work was supported by the Swiss National Science Foundation and by the NCCR Program MaNEP sponsored by the Swiss National Science Foundation. Ames Lab is operated for the U.S. Department of Energy by Iowa State University under Contract No. W-7405-Eng-85. The work at the Ames Lab was supported by the Director of Energy Research, Office of Basic Energy Sciences.
*Email address: dicastro@physik.unizh.ch

${ }^{1}$ J. Nagamatsu, N. Nakagawa, T. Muranaka, Y. Zenitani, and J. Akimitsu, Nature (London) 410, 63 (2001).

${ }^{2}$ S. L. Bud'ko, G. Lapertot, C. Petrovic, C. E. Cunningham, N. Anderson, and P. C. Canfield, Phys. Rev. Lett. 86, 1877 (2001).

${ }^{3}$ D. G. Hinks, H. Claus, and J. D. Jorgensen, Nature (London) 411, 457 (2001).

${ }^{4}$ D. Di Castro, S. Agrestini, G. Campi, A. Cassetta, M. Colapietro, A. Congeduti, A. Continenza, S. De Negri, M. Giovannini, S. Massidda, M. Nardone, A. Pifferi, P. Postorino, G. Profeta, A. Saccone, N. L. Saini, G. Satta, and A. Bianconi, Europhys. Lett. 58, 278 (2002).

${ }^{5}$ A. F. Goncharov and V. V. Struzhkin, Physica C 385, 117 (2003).

${ }^{6}$ F. Bouquet, R. A. Fisher, N E. Phillips, D. G. Hinks, and J. D. Jorgensen, Phys. Rev. Lett. 87, 047001 (2001).
${ }^{7}$ A. Y. Liu, I. I. Mazin, and J. Kortus, Phys. Rev. Lett. 87, 087005 (2001).

${ }^{8}$ H. J. Choi, D. Roundy, H. Sun, M. L. Cohen, and S. G. Louie, Nature (London) 418, 758 (2002).

${ }^{9}$ R. S. Gonnelli, D. Daghero, G. A. Ummarino, V. A. Stepanov, J. Jun, S. M. Kazakov, and J. Karpinski, Phys. Rev. Lett. 89, 247004 (2002).

${ }^{10}$ P. Szabo, P. Samuely, J. Kačmarčik, T. Klein, J. Marcus, D. Fruchart, S. Miraglia, C. Marcenat, and A. G. M. Jansen, Phys. Rev. Lett. 87, 137005 (2001).

${ }^{11}$ S. Souma, Y. Machida, T. Sato, T. Takahashi, H. Matsui S.-C. Wang, H. Ding, A. Kaminski, J. C. Campuzano, S. Sasaki, and K. Kadowaki, Nature (London) 423, 65 (2003).

${ }^{12}$ M. Angst, R. Puzniak, A. Wisniewski, J. Jun, S. M. Kazakov, J. Karpinski, J. Roos, and H. Keller, Phys. Rev. Lett. 88, 167004 
(2002).

${ }^{13}$ A. S. Alexandrov, Physica C 363, 231 (2001).

${ }^{14}$ E. Cappelluti, S. Ciuchi, C. Grimaldi, L. Pietronero, and S. Strässler, Phys. Rev. Lett. 88, 117003 (2002).

${ }^{15}$ G.-M. Zhao, New J. Phys. 4, 3.1 (2002).

${ }^{16}$ A. S. Alexandrov and N. F. Mott, Int. J. Mod. Phys. B 8, 2075 (1994)

${ }^{17}$ C. Grimaldi, E. Cappelluti, and L. Pietronero, Europhys. Lett. 42, 667 (1998).

${ }^{18}$ A. Bussmann-Holder, R. Micnas, and A. R. Bishop, Philos. Mag. 84, 1257 (2004).

${ }^{19}$ G. M. Zhao and D. E. Morris, Phys. Rev. B 51, 16487 (1995).

${ }^{20}$ Guo-meng Zhao, M. B. Hunt, H. Keller, and K. A. Müller, Nature (London) 385, 236 (1997).

${ }^{21}$ G. M. Zhao, K. Conder, H. Keller, and K. A. Müller, J. Phys.: Condens. Matter 10, 9055 (1998).

${ }^{22}$ J. Hofer, K. Conder, T. Sasagawa, Guo-meng Zhao, M. Willemin, H. Keller, and K. Kishio, Phys. Rev. Lett. 84, 4192 (2000).

${ }^{23}$ Guo-meng Zhao, H Keller, and K Conder J. Phys.: Condens. Matter 13, R569 (2001).

${ }^{24}$ R. Khasanov, A. Shengelaya, K. Conder, E. Morenzoni, I. M. Savić, and H. Keller, J. Phys.: Condens. Matter 15, L17 (2003).

${ }^{25}$ R. Khasanov, D. G. Eshchenko, H. Luetkens, E. Morenzoni, T. Prokscha, A. Suter, N. Garifianov, M. Mali, J. Roos, K. Conder, and H. Keller, Phys. Rev. Lett. 92, 057602 (2004).

${ }^{26}$ R. Khasanov, A. Shengelaya, E. Morenzoni, M. Angst, K. Conder, I. M. Savic, D. Lampakis, E. Liarokapis, A. Tatsi, and H. Keller, Phys. Rev. B 68, 220506(R) (2003).

${ }^{27}$ B. Pümpin, H. Keller, W. Kündig, W. Odermatt, I. M. Savić, J. W. Schneider, H. Simmler, P. Zimmermann, E. Kaldis, S. Rusiecki, Y. Maeno, and C. Rossel, Phys. Rev. B 42, 8019 (1990).

${ }^{28}$ R. W. Shaw, D. E. Mapother, and D. C. Hopkins, Phys. Rev. 121, 86 (1961).
${ }^{29}$ R. A. Ribeiro, S. L. Bud'ko, C. Petrovic, and P. C. Canfield, Physica C 385, 16 (2003).

${ }^{30}$ Random flux pinning near the lower critical field $H_{c 1}$ may induce distortion of the VL, giving rise to a field dependent $\sigma$ which affects the value of $\lambda$.

${ }^{31}$ Ch. Niedermayer, C. Bernhard, T. Holden, R. K. Kremer, and K. Ahn, Phys. Rev. B 65, 094512 (2002).

${ }^{32}$ K. Ohishi, T. Muranaka, j. Akimitsu, A. Koda, W. Higemoto, and R. Kadono, J. Phys. Soc. Jpn. 72, 29 (2003)

${ }^{33}$ R. Cubitt, M. R. Eskildsen, C. D. Dewhurst, J. Jun, S. M. Kazakov, and J. Karpinski, Phys. Rev. Lett. 91, 047002 (2003).

${ }^{34}$ L. Lyard, P. Szabo, T. Klein, J. Marcus, C. Marcenat, K. H. Kim, B. W. Kang, H. S. Lee, and S. I. Lee, Phys. Rev. Lett. 92, 057001 (2004).

${ }^{35}$ M. Angst, D. Di Castro, D. G. Eshchenko, R. Khasanov, S. Kohout, I. M. Savić, A. Shengelaya, S. L. Bud'ko, P. C. Canfield, J. Jun, J. Karpinski, S. M. Kazakov, R. A. Ribeiro, and H. Keller (unpublished).

${ }^{36}$ See, e.g., M. H. S. Amin, M. Franz, and I. Affleck, Phys. Rev. Lett. 84, 5864 (2000).

${ }^{37}$ W. Barford and J. M. F. Gunn, Physica C 156, 515 (1998).

${ }^{38}$ V. G. Kogan, Phys. Rev. B 66, 020509 (2002).

${ }^{39}$ A. A. Golubov, A. Brinkman, O. V. Dolgov, J. Kortus, and O. Jepsen, Phys. Rev. B 66, 054524 (2002).

${ }^{40}$ M. Tinkham, Introduction to Superconductivity (Krieger, Malabar, 1975).

${ }^{41}$ A. V. Sologubenko, J. Jun, S. M. Kazakov, J. Karpinski, and H. R. Ott, Phys. Rev. B 66, 014504 (2002).

${ }^{42}$ F. Bouquet, Y. Wang, I. Sheikin, T. Plackowski, A. Junod, S. Lee, and S. Tajima, Phys. Rev. Lett. 89, 257001 (2002).

${ }^{43}$ D. Di Castro, R. Khasanov, C. Grimaldi, J. Karpinski, S. M. Kazakov, and H. Keller (unpublished). 